\title{
Ptychographic Imaging in an Aberration Corrected STEM
}

\author{
Andrew R. Lupini ${ }^{1,3}$, Miaofang Chi $^{2}$, Sergei V. Kalinin ${ }^{2,3}$, Albina Y. Borisevich ${ }^{1,3}$, Juan Carlos Idrobo ${ }^{2}$, \\ Stephen Jesse ${ }^{2,3}$. \\ ${ }^{1}$ Materials Science \& Technology Division, Oak Ridge National Laboratory, Oak Ridge, TN 37831 \\ ${ }^{2}$ Center for Nanophase Materials Sciences, Oak Ridge National Laboratory, Oak Ridge, TN 37831 \\ ${ }^{3}$ Institute for Functional Imaging of Materials, Oak Ridge National Laboratory, Oak Ridge, TN 37831
}

One of the traditional limitations of electron microscopy is that although the phase and the amplitude of the transmitted electron beam both contain information about the sample, the detectors are only able to record the intensity. Furthermore most Scanning Transmission Electron Microscope (STEM) imaging modes rely on integrating the transmitted beam over some range of scattering angles. For example, the high-angle annular dark field (HAADF) mode provides 'Z-contrast' images because the amount of scattering to high angles depends on the atomic number. The STEM is also able to record bright field (BF) images which, through the principle of reciprocity, are equivalent to conventional TEM images. However, in order to obtain a sufficiently coherent phase-contrast image, the collection angle for BFSTEM has to be much smaller than the convergence angle, meaning that BF-STEM is expected to be much less efficient than TEM. One solution to this predicament is to use a pixelated detector to record the scattering to every angle as a function of probe position. The resulting four dimensional (4D) data set consisting of a 2D image or 'Ronchigram' for each probe position in a $2 \mathrm{D}$ array provides equivalent information to recording the set of TEM images as a function of tilt angle and can be used to reconstruct the complex exit wavefunction [1]. The experimental configuration is illustrated in Figure 1.

Other imaging modes are available in STEM, including differential phase contrast (DPC) which has been shown to allow imaging of polarization at atomic resolution [2] and annular bright field (ABF) [3] which is particularly useful for locating light atoms. These images can also be recorded on a pixelated detector, with the advantage that the particular range of scattering to integrate over can be chosen after the experiment in post-processing and several images can be formed simultaneously (Figure 2). Collecting all of the transmitted beam also allows highly efficient phase contrast images to be recorded and to apply filters that would not be possible with a single detector [4].

We have recorded data sets on both conventional CCDs and on a direct electron detector able to detect single electrons [5] attached to an aberration corrected FEI Titan operating at $300 \mathrm{kV}$. A set of example images generated from such a data set are shown in Figure 2. Images were collected on a DE-12 camera (Direct Electron, LP, San Diego, CA), equipped with a 4096 x 3072 pixels Direct Detection Device $(\mathrm{DDD} \AA)$ sensor. Each exposure was acquired with continuous frame streaming at $768 \times 768$ pixels binned $\mathrm{x} 2$ at 300 frames per second. We have developed a custom beam control system to synchronize the STEM scanning to the Ronchigram acquisition. This system is able to synchronize to a variety of triggers at the hardware level, to scan unconventional scan patterns, and to scan at a variable speed within a single image [6]. Another common use for the electron Ronchigram is to accurately measure aberrations in order to allow efficient alignment of an aberration corrector. These methods usually need an amorphous region or special tuning sample, even if the sample of interest is crystalline. However, the 4D data set also contains equivalent information and routes to extract aberrations and other imaging data will be discussed. [7] 
References:

[1] J. M. Rodenburg, B. C. McCallum and P. D. Nellist, Ultramicroscopy, 48 (1993), p. 304.

[2] N. Shibata et al, Nature Physics, 8 (2012), p. 611.

[3] R. Ishikawa, et al, Nature Materials 10 (2011), p. 278.

[4] T.J. Pennycook, et al, Ultramicroscopy (2014), in press.

[5] A.-C. Milazzo, et al. Ultramicroscopy 104 (2005), p. 152.

[6] S. Jesse, et al, these proceedings.

[7] Research supported by Division of Materials Sciences and Engineering Division, Office of Basic

Energy Sciences, U.S. DOE (ARL, AYB) and by Oak Ridge National Laboratory's Center for

Nanophase Materials Sciences (CNMS), which is sponsored by the Scientific User Facilities Division, Office of Basic Energy Sciences, U.S. Department of Energy (MC, SVK, JCI, SJ). The authors acknowledge technical assistance from Liang Jin and Benjamin Bammes of Direct Electron, and Hans Christen and Christianne Beekman for providing samples.

(A)

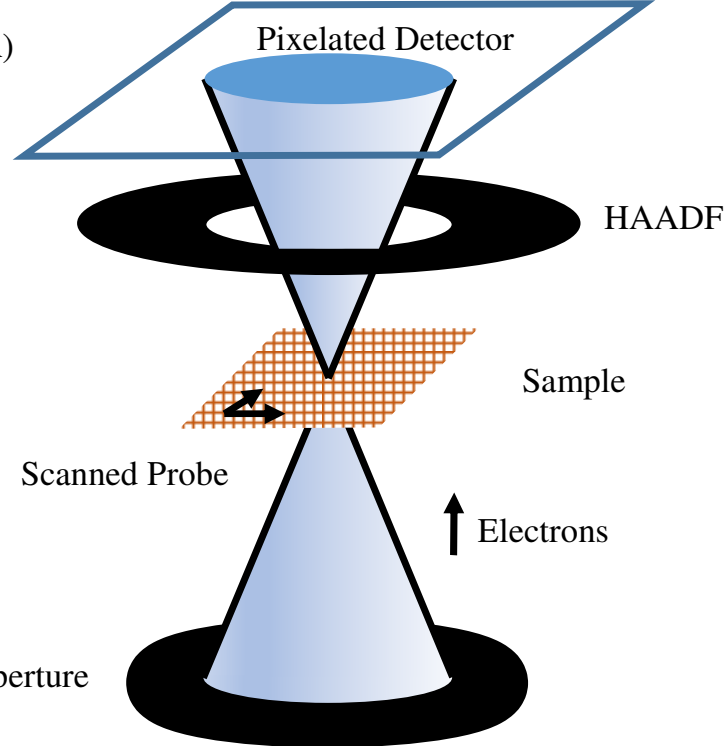

(B)

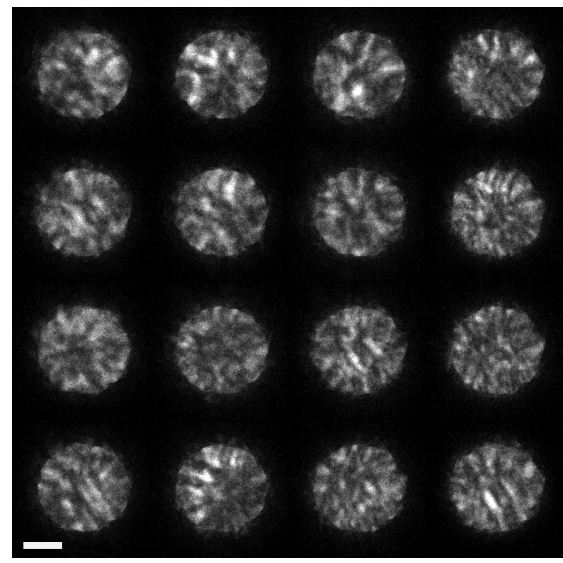

(C)

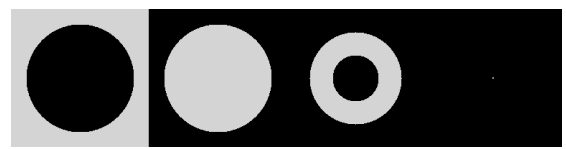

Figure 1. (A) Schematic illustration of the data acquisition scheme. The pixelated detector records the transmitted intensity as a 2D image for each probe position on the sample. (B) Typical Ronchigrams from a $\mathrm{La}_{0.67} \mathrm{Sr}_{0.33} \mathrm{MnO}_{3} / \mathrm{SrTiO}_{3}$ sample. Scale bar is $30 \mathrm{mrad}$. (C) Example detector configurations (same scale).
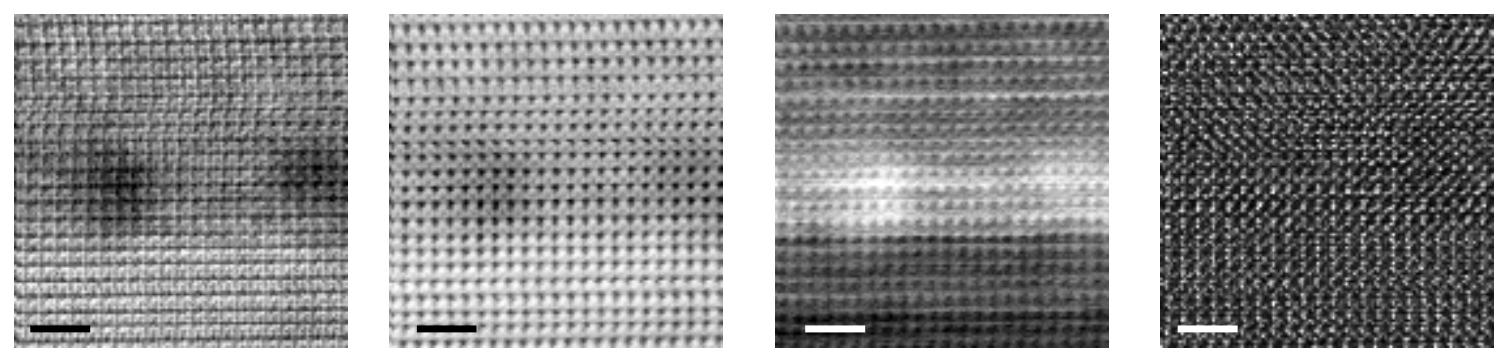

Figure 2. Integrating the Ronchigram data set over a range of angles is used to form multiple images of the sample from Figure 1(B) using the detector configurations in (C): From left to right, a low-angle dark field image, a BF image with a large detector, an annular BF image, and a BF image with a small detector. Scale bar is $2 \mathrm{~nm}$. 